\title{
Influencia del factor de crecimiento fibroblástico 2 en células madre in vitro
}

\author{
Influence of fibroblast growth factor 2 on stem cells in vitro
}

Catalina Rubio-Vargas ${ }^{1,3^{*}}$, Jessica Alcázar ${ }^{2,3}$, Liliana Francis-Turner ${ }^{2,3}$

\begin{abstract}
Resumen
El interés y la necesidad de estudiar las células madre en terapias regenerativas han aumentado en los últimos años debido a su capacidad de proliferación y diferenciación hacia múltiples linajes. Desafortunadamente, el número de células demandado para un trasplante satisfactorio es mayor al que se logra extraer directamente del paciente, por lo que se deben realizar cultivos in vitro de células madre. Sin embargo, con el tiempo las células de estos cultivos se vuelven senescentes, disminuyendo así su número de divisiones celulares y su capacidad proliferativa. Para solucionar esta problemática se han propuesto factores de crecimiento como agentes potenciadores de la proliferación celular. Entre estos se encuentra el factor de crecimiento fibroblástico 2, que al agregarse al medio podría promover la proliferación y aumentar el tiempo de vida de las células en cultivo. En la siguiente revisión se recopila información sobre la biología de este factor de crecimiento y el tipo de señalización que utiliza, al igual que sus aplicaciones en terapia regenerativa y su efecto en la proliferación celular y senescencia.
\end{abstract}

Palabras claves: FGF-2, proliferación celular, senescencia, terapia celular.

\begin{abstract}
The interest and need to study stem cells in regenerative therapies has increased in recent years due to their ability to proliferate and differentiate into multiple lineages. Unfortunately, the number of cells needed for a successful transplant is greater than that obtained directly from the patient, so in vitro cultures of stem cells must be done. However, over time the cells in these cultures become senescent, decreasing their number of cellular divisions and their proliferative capacity. To solve this problem, different growth factors have been proposed as promoting agents for cell proliferation. Among these is the fibroblast growth factor 2 which, when added to the medium, could promote proliferation and increase the life time of the cells in the culture. The following review compiles information about the biology of this growth factor and the type of signaling it uses, as well as its applications in regenerative therapy and its effect on cell proliferation and senescence.
\end{abstract}

Keywords: FGF-2, cell proliferation, senescence, cellular therapy.

\footnotetext{
1. Programa de Biología, Facultad de Ciencias, Universidad del Tolima, Ibagué, Colombia.

2. Facultad de Ciencias, Universidad del Tolima, Ibagué, Colombia.

3. Grupo de modelos experimentales para las ciencias zoohumanas, Facultad de Ciencias, Universidad del Tolima, Ibagué, Colombia.

* Autor de correspondencia: <acrubiov@ut.edu.co>
} 


\section{INTRODUCCIÓN}

La utilización de Células Madre (CM) en ingeniería de tejidos ha aumentado en las últimas décadas debido a que estas células representan una fuente autóloga para posibles tratamientos de enfermedades como Alzheimer, Parkinson, cáncer y diabetes, entre otras (Carvalho et al. 2013, Espinoza et al. 2016, Hernández et al. 2011, Quimby y Borjesson 2018). Sin embargo, el número de CM extraídas directamente del paciente es insuficiente para un trasplante exitoso, por lo que, generalmente, se requiere realizar una expansión in vitro (Brizuela et al. 2013, Yang 2018). Desafortunadamente, se ha observado que después de un prolongado tiempo en cultivo las células pierden progresivamente su capacidad proliferativa y su función de auto-regeneración, ocasionando alteraciones en su morfología, lo que dificulta su uso en terapia regenerativa (Apel et al. 2009, Baker et al. 2015, Richardson et al. 2016). Para mantener el funcionamiento adecuado de las CM sin alterar su respectivo fenotipo se ha propuesto el acondicionamiento del medio de cultivo con factores que promueven la proliferación y benefician el metabolismo celular, inhibiendo la muerte y el envejecimiento de las células (Gharibi y Hughes 2012, Inoue et al. 2007).

A nivel in vitro, Mckay et al. (1989) estimularon significativamente la división celular en explantes epiteliales mediante la adición de factores de crecimiento fibroblástico (FGF). Posteriormente, Taupin et al. (2000) demostraron que la influencia de la adición de factores como el FGF-2, contribuye al aumento de la tasa proliferativa del cultivo celular. De este modo, la implicación de FGF en un cultivo in vitro puede proporcionar un ambiente óptimo para la expansión celular que estimula la capacidad de diferenciación de las CM.

Con el propósito de evidenciar el efecto que tiene la aplicación de FGF-2 en la supervivencia del cultivo de CM, se presenta la siguiente revisión de tema, realizada mediante una búsqueda bibliográfica en bases de datos como Scopus, Scielo, Science Direct y PubMed, utilizando palabras estratégicas como: "FGF2", "terapia celular", "células madre", "stem cells", "cell proliferation". Los artículos fueron seleccionados de acuerdo con su importancia y aplicabilidad para el tema tratado.

\section{BIOLOGÍA DEL FACTOR DE CRECIMIENTO FI- BROBLÁSTICO 2 (FGF-2)}

Los FGF son un conjunto de proteínas y péptidos bioreguladores que intervienen en el desarrollo de diferentes tejidos. Esta familia está compuesta por un grupo de 23 miembros identificados en vertebrados e invertebrados los cuales varían en peso molecular de 17 a 34 kD (Kurosu et al. 2006, Zhang y Li 2016).

El FGF-2, al igual que los otros miembros de la familia de FGF, posee una secuencia conservada (16$65 \%$ ), la cual tiene un dominio de 120 aminoácidos que interactúan con receptores específicos (FGFR) que presentan tres dominios extracelulares tipo inmunoglobulina y un dominio intracelular tirosina quinasa (Korsensky y Ron 2016).

De acuerdo con su funcionalidad este factor presenta señalización paracrina y está implicado en aspectos del desarrollo celular que incluyen la proliferación, crecimiento y diferenciación; también actúan sobre diferentes tipos de células con el fin de regular funciones fisiológicas como la angiogénesis, el crecimiento celular, el desarrollo embrionario, la regulación metabólica, la migración celular y la reparación de tejidos (Markan y Potthoff 2016, Yonemitsu et al. 2019).

\section{RUTAS DE SEÑALIZACIÓN CELULAR IMPLICA- DAS EN PROLIFERACIÓN CELULAR}

Los FGF realizan su acción proliferativa mediante la activación de diferentes vías de señalización celular. Estos pueden tener interacciones directas con receptores específicos de membrana (FGF-R) o con otras proteínas celulares ligadas a receptores HSPG, que llevan a la activación de receptores tirosina quinasa y extienden cascadas de señalización de la vía RAS (Fernandes-Freitas y Owen 2015, Naugler et al. 2015).

El FGF-2 presenta señalización paracrina, es decir, se encarga de realizar enlaces de alta afinidad con receptores HSPG que sirven como cofactores para la interacción con los FGF-R (Coutu et al. 2011), y 
estos a su vez activan cascadas de señalización como RAS/RAF/MAPK, PI3K/AKT y PLC $\gamma$, cumpliendo un papel fundamental en el desarrollo de órganos como el corazón, el cerebro, el músculo y los riñones (El Agha et al. 2016).

\section{FGF-2 Y SU IMPLICACIÓN EN LA SUPERVIVEN- CIA DE CÉLULAS MADRE}

La utilización de las CM en terapia regenerativa ha aumentado en la última década gracias a su capacidad de diferenciarse hacia varios tejidos (Cui et al. 2017); lo anterior se evidencia en una búsqueda en el banco de datos del Instituto Nacional de Salud de Estados Unidos (https://www.clinicaltrials.gov); utilizando el término Mesenchymal stem cell, el 10-062019 se encontraron 757 estudios clínicos al respecto, variando entre trasplante para enfermedades pulmonares y diabetes (Can et al. 2017), reconstrucción del epitelio corneal (Ziaei et al. 2017), tratamientos en Alzheimer (Wang et al. 2018), etc.

No obstante, a nivel in vitro, las CM necesitan ser estimuladas para prevenir el envejecimiento prematuro, también conocido como senescencia replicativa (de Magalhães y Passos 2018, Hong et al. 2019). Debido a que con el aumento del número de pasajes las características proliferativas y el potencial de diferenciación de las CM se van perdiendo, su uso en terapia celular se ve afectado (Guo et al. 2010, Hong et al. 2019, Wang et al. 2015, Zhai et al. 2019). En este proceso, el FGF-2 juega un papel estratégico al desencadenar la activación de cascadas intracelulares, responsables de la proliferación y diferenciación que potencian la vida del cultivo (Lai et al. 2011).

La funcionalidad de los FGF es bastante extendida, dada su influencia en el desarrollo embrionario (Dermargos y Armelin 2007), encargándose de la migración y diferenciación celular que da lugar a la formación de órganos (Ahn et al. 2009, Chen et al. 2019, Novais et al. 2019, Ornitz y Itoh 2001). En la etapa adulta, los FGF son mitógenos potenciales en la cicatrización de heridas y angiogénesis; además, algunos FGF actúan manteniendo la homeostasis de ácidos biliares, glucosa y fosfato (Brewer et al. 2016).
Este factor es descrito como un importante mitógeno que contribuye a preservar la multipotencialidad de las CM. Adicionalmente, Coutu et al. (2011) sugirieron que la adición de FGF-2 en el medio de cultivo podría regular el estado de senescencia a través de los receptores FGF-R1/2. Nowwarote et al. (2015) registraron que el FGF-2 promueve la proliferación celular mediante la activación de la vía PI3K/AKT, manteniendo el cultivo celular con sus características de auto renovación.

\section{APLICACIÓN DEL FGF-2 A UN CULTIVO CELU- LAR}

Diferentes autores mencionan que la adición de FGF2 promueve la proliferación celular. Bianchi et al. (2014) evidenciaron que un cultivo de CM extraídas de médula ósea de donantes sanos con edades entre 3 a 49 años, cultivadas en una densidad de 2 a $5 \times 10^{6}$ células en 100 mm en medio Ham's F-12 enriquecido con suero fetal bovino (SFB) al $10 \%$ y $1 \mathrm{ng} / \mathrm{ml}$ de FGF-2, logró incrementar gradualmente la formación de telómeros, mientras que en los cultivos control carentes de FGF-2, la longitud de los telómeros en las células disminuyó constantemente. Adicionalmente evidenciaron que el FGF-2 aumentó la tasa de proliferación celular y retrasó el cambio en la morfología de estas, el cual se vio influenciado en función de la edad. La adición de FGF-2 indujo también una pérdida del potencial condrogénico de estas células.

Otros autores como Zheng et al. (2004), evaluaron la capacidad de este mismo factor en la manutención de CM de precursor neural encontradas en la zona subventricular del cerebro de ratón, determinando que este factor contribuye no solo a la proliferación neural sino que también a la astroglial y a la inducción de progenitores oligodendrogliales.

Estos estudios son apoyados por Wang et al. (2015), quienes aislaron células madre de médula ósea del panda gigante (Ailuropoda melanoleuca) e investigaron el potencial de proliferación y diferenciación de estas células a nivel in vitro en adición del FGF-2, el cual ha demostrado desempeñar un papel fundamental en el mantenimiento, diferenciación y aumento de la longitud de telómeros. Durante el procedimiento, 
las células fueron cultivadas a $37{ }^{\circ} \mathrm{C}$, en un medio enriquecido con $10 \mathrm{ng} / \mathrm{ml}$ de factor de crecimiento epidérmico. Además, se realizaron diferentes tratamientos donde variaba la concentración de FGF-2, siendo estos: $0,1,5,10 \mathrm{ng} / \mathrm{ml}$; los autores encontraron una mayor capacidad proliferativa en las células enriquecidas con $5 \mathrm{ng} / \mathrm{ml}$ de FGF-2, demostrando además que la vía de señalización ERK es fundamental en este proceso.

Posiblemente el FGF-2 disminuye el proceso de senescencia a nivel in vitro $\mathrm{y}$ el envejecimiento in vivo, donde los telómeros se van acortando debido a la replicación unidireccional de las polimerasas del ADN. De esta forma, Bianchi et al. (2003) indicaron que el FGF-2 selecciona un subconjunto de células progenitoras con telómeros más largos que, al cultivarse en baja densidad y la presencia de este factor, incrementan la vida útil de las CM; adicionalmente, este factor demostró ser fundamental en el proceso de autorrenovación celular. Sin embargo, aunque Bianchi et al. (2003) y Wang et al. (2018) describen su potencial de diferenciación condrogénico, la dosis determinada para una proliferación exitosa varía de 1 a $5 \mathrm{ng} / \mathrm{ml}$. Por ende, es primordial conocer otros registros de dosis de este factor junto a sus interacciones con otros factores de crecimiento.

Eom et al. (2014) determinaron la capacidad proliferativa de diferentes FGF, extrayendo $\mathrm{CM}$ de médula ósea y adicionando factores de crecimiento tales como: FGF-2 (1 ng/ml), FGF-4 (10 ng/ml) y HGF (10 $\mathrm{ng} / \mathrm{ml}$ ) al medio de cultivo, donde evidenciaron el aumento en la proliferación celular en aquellos tratamientos que contenían FGF 2. También se reportó que hay una activación de la vía ERK por medio del FGF-2, sugiriendo que este factor podría suprimir el proceso de senescencia celular.

Recientemente, Lee et al. (2018) sometieron experimentalmente a las $\mathrm{CM}$ de médula ósea humana a hipoxia y aplicaron $5 \mathrm{ng} / \mathrm{ml}$ de FGF-2, diferenciando los siguientes tratamientos: normoxia / FGF-2 (-), normoxia / FGF-2 (+), hipoxia / FGF-2 (-) e hipoxia / FGF-2 (+). La hipoxia fue inducida colocando las células en una incubadora que contenía $1 \%$ de $\mathrm{O}_{2}$ en $37{ }^{\circ} \mathrm{C}$; los autores denominaron normoxia cuando la incubadora contenía $21 \%$ de $\mathrm{O}_{2}$. Como resultado demostraron que las condiciones de hipoxia y normoxia no eran significativas en la proliferación celular, como sí lo era la presencia del FGF-2, donde aumentó significativamente el número de colonias en el cultivo.

\section{EFECTO DEL FGF-2 EN UN CO-CULTIVO DE CM}

Coutu et al. (2011) demostraron que enriquecer el cultivo con $4 \mathrm{ng}$ de FGF aumenta la proliferación celular y que al encontrarse en un co-cultivo con adipocitos, estimula la diferenciación hacia este linaje celular. Por otro lado se ha demostrado que en un cultivo con FGF-2 y durante un periodo de 10 a 50 días se evidencia una notable disminución de células senescentes, posiblemente debido a que el FGF-2 además de estimular la proliferación actúa dando una señal negativa a la expresión de la proteína TGF- $\beta 2$, responsable de la senescencia celular (Ito et al. 2007).

Nawrocka et al. (2017) aislaron CM de tejido adiposo subcutáneo de donantes sanos y pacientes con diabetes tipo 2, las cuales fueron mantenidas en medio DMEM al cual se añadió $1 \%$ de antibiótico y $10 \%$ de SFB a $37{ }^{\circ} \mathrm{C}$, y evaluaron la acción del FGF-2 en dosis de 5 y $10 \mathrm{ng} / \mathrm{ml}$, el cual se agregó directamente al cultivo y fue revisado después de 7 días. Como resultado se obtuvo que la proliferación celular disminuyó constantemente, aunque la dosis de FGF-2 de $5 \mathrm{ng} / \mathrm{ml}$ indujo la proliferación de las CM de tejido adiposo. Adicionalmente en los cultivos tratados se observó un aumento de células alargadas con un núcleo central, las cuales, en 7 días, formaron múltiples capas; además en el cocultivo de CM extraídas de pacientes con diabetes tipo 2 y CM de pacientes sanos, el FGF-2 aumentó la tasa de proliferación celular y disminuyó el efecto de senescencia y apoptosis.

\section{DISCUSIÓN}

Características como la capacidad proliferativa, la regeneración de tejidos y la plasticidad fenotípica, han hecho que las CM sean postuladas como una fuente potencial para la terapia regenerativa (Can et al. 2017, Huang et al. 2011, Katsares et al. 2009). Sin embargo, para un trasplante exitoso es necesario 
obtener suficiente cantidad de células, por lo que es fundamental un proceso de expansión in vitro; no obstante, su potencial de diferenciación y crecimiento celular se ve comprometido después de un largo tiempo en cultivo, dando lugar a un proceso de detención celular denominado senescencia (Chen et al. 2015, Cui et al. 2017, Drela et al. 2014). El FGF-2 es propuesto como un factor capaz de atenuar procesos como la senescencia y el estrés oxidativo (Dolivo et al. 2016). Aunque entre los estudios expuestos durante esta revisión se discriminan diferentes concentraciones del FGF-2, al analizar las dosis tratadas se puede considerar que el mejor resultado en la manutención del cultivo se logró aplicando 5 ng/ml de FGF-2. A pesar de que en estos cultivos las células muestran un aumento en la proliferación celular, después de un largo tiempo in vitro, entran en un estado de senescencia celular o límite de Hayflick (Meng et al. 2017). Ito et al. (2007) reportan que la estimulación del FGF-2 en un cultivo de CM al día 50 presentaba el $40 \%$ de células senescentes, no obstante, en los tratamientos control (en ausencia de FGF-2) el porcentaje de células senescentes a los 50 días, fue $57,6 \%$, por lo que es posible concluir que este factor de crecimiento logra atenuar el proceso de senescencia celular, prolongando el tiempo de vida de las células.

Rubio-Vargas et al. (2018) encontraron que la adición de FGF-2 en el cultivo disminuyó la producción de especies reactivas de oxígeno (ROS), evidenciando que este factor no solo contribuye al mantenimiento del cultivo, sino que también promueve la regulación de ROS intracelulares. Cabe resaltar que las ROS son los principales mediadores de senescencia celular durante la expansión in vitro; además de los daños causados por el estrés oxidativo como lo son el daño de ADN y la ruptura de membrana, se ha demostrado que las ROS inducen a la diferenciación de las células, lo que limita su utilización a nivel in vitro.

Por lo anterior, el FGF-2 podría actuar como un factor antioxidante, reduciendo la concentración de ROS y, en consecuencia, el estado de senescencia celular. Sin embargo, para corroborar esta hipótesis, es necesario realizar un estudio de señalización de vías celulares. Pese a los resultados positivos que se obtienen de la aplicación del FGF-2 en el medio, algunos estudios como el de Preda et al. (2015), quienes cultivaron $\mathrm{CM}$ con medio enriquecido con factor de crecimiento transformante beta 1 (TGFB1) (10 ng/ml) y FGF-2 (50 ng/ml), demostraron que aunque la aplicación del FGF-2 promueve un aumento en la proliferación celular, presenta un efecto citotóxico al producir un colapso en el índice celular.

Estos resultados son contradictorios ya que otros autores como Sah et al. (2019) han utilizado el FGF-2 en la elaboración de matrices sintéticas que permiten conservar el fenotipo inicial de las CM de músculo esquelético; las células cultivadas en dicha matriz fueron aisladas de los músculos de las extremidades posteriores de ratones ICR (Institute of Cancer Research) de 6 a 8 semanas de edad y sembradas en medio de crecimiento (Ham-F10 con suero de caballo al $20 \%, 1 \%$ penicilina-estreptomicina, $5 \mathrm{ng} / \mathrm{ml} \mathrm{de}$ FGF2 humano, en una incubadora con $5 \%$ de $\mathrm{CO}_{2}$ a $37^{\circ} \mathrm{C}$ ), registrando un estado indiferenciado de las células cultivadas en dicha matriz y demostrando una fuerte capacidad de proliferación. Este estudio fue corroborado por los análisis de Endo et al. (2019). Estos estudios informan que el acondicionamiento del medio de cultivo con FGF-2 promueve la proliferación de CM mediante la adición de $10 \mathrm{ng} / \mathrm{ml}$ de FGF-2 a partir del pasaje 0, debido a que el FGF2 mejora el potencial mitótico y no conduce a las células a diferenciación, regulando la expresión del gen SOX2.

\section{CONCLUSIONES}

Los FGF cumplen variadas funciones en el desarrollo embrionario como mitógenos y proteínas señalizadoras de proliferación, migración y diferenciación celular; en la etapa adulta, los FGF son esenciales como moléculas homeostáticas encargadas de regular diferentes procesos metabólicos, como la producción de ácido biliar, fosfatasa y glucosa en el individuo.

Diferentes estudios han registrado sobre la acción del FGF-2 en la proliferación celular a nivel in vitro características que los postulan como elementos claves en la terapia regenerativa y la ingeniería de tejidos. Adicionalmente se ha demostrado que el FGF-2 puede retrasar el tiempo de senescencia celular en el cultivo y posiblemente puede disminuir 
la cantidad de ROS en la célula. Sin embargo, las concentraciones analizadas varían desde $1 \mathrm{ng}$ hasta $10 \mathrm{ng}$, encontrándose hasta el momento como mejor opción la concentración de $5 \mathrm{ng} / \mathrm{ml}$, la cual facilita la proliferación celular sin promover una diferenciación específica de la célula; además, aún son pocos los estudios a nivel molecular que se han realizado sobre las vías de señalización de los FGF-2 en las CM, y no se tiene claridad sobre los mecanismos implicados en este proceso, por lo que se requiere realizar más estudios del FGF-2 para comprender su biología y sus diferentes vías de acción.

Estudios recientes reportan que el FGF-2 es un mediador del estado pluripotente de las células, el cual influencia la activación de la ruta de señalización MAPK, y, a partir del enriquecimiento del medio con diferentes tratamientos de FGF-2 (100 ng/ml; $5 \mathrm{ng} / \mathrm{ml}$ y sin adición de FGF-2), concluyeron que las CM requieren la presencia de este factor de manera exógena para mantener la auto-renovación y la capacidad de diferenciarse; además, mediante la detección de proteínas se encontró que la ruta MAPK es activada por FGF-2 y permite a las células estar en su estado indiferenciado (Haghighi et al. 2018), por lo que se necesita conocer qué rutas de señalización están implicadas en los procesos de proliferación cuando se utiliza FGF-2 de manera exógena.

Finalmente, se requiere conocer el comportamiento del FGF-2 en cultivo en conjunto con otros compuestos para potenciar la vida útil del cultivo; al respecto, Park et al. (2018) compararon el efecto del FGF2 y el del selenio, (previamente descrito como un supresor de senescencia celular) en conjunto y por separado, y determinaron en $5 \mathrm{ng} / \mathrm{ml}$ la concentración óptima de selenio y $4 \mathrm{ng} / \mathrm{ml}$ la de FGF-2, debido a que estas concentraciones registraron una mayor tasa de proliferación. Por lo anterior, el FGF-2 puede promover sus funciones proliferativas con el uso en conjunto de otros compuestos, mejorando los efectos en terapia de $\mathrm{CM}$ en la regeneración de tejidos.

\section{CONFLICTO DE INTERESES}

Los autores declaran no tener conflictos de intereses.

\section{REFERENCIAS}

Ahn H-J, Lee W, Kwack K, Kwon Y. 2009. FGF2 stimulates the proliferation of human mesenchymal stem cells through the transient activation of JNK signaling. FEBS Letters, 583(17): 2922-2926. doi:10.1016/j.febslet.2009.07.056

Apel A, Groth A, Schlesinger S, Bruns H, Schemmer P, Büchler MW, Herr I. 2009. Suitability of human mesenchymal stem cells for gene therapy depends on the expansion medium. Experimental Cell Research, 315(3): 498-507. doi:10.1016/j.yexcr.2008.11.013

Baker N, Boyette L, Tuan-R S. 2015. Characterization of bone marrow-derived mesenchymal stem cells in aging. Bone, 70: 37-47. doi:10.1016/j.bone.2014.10.014

Bianchi G, Banfi A, Mastrogiacomo M, Notaro R, Luzzatto L, Cancedda R, Quarto R. 2003. Ex vivo enrichment of mesenchymal cell progenitors by fibroblast growth factor 2. Experimental Cell Research, 287(1): 98-105. doi:10.1016/s0014-4827(03)00138-1

Brewer JR, Mazot P, Soriano P. 2016. Genetic insights into the mechanisms of Fgf signaling. Genes and Development, 30(7): 751-771. doi:10.1101/gad.277137.115

Brizuela C, Galleguillos S, Carrión F, Cabrera C, Luz P, Inostroza C. 2013. Aislación y caracterización de células madre mesenquimales provenientes de pulpa y folículo dentario humano. International Journal of Morphology, 31(2): 739-746. doi:10.4067/S0717-95022013000200063

Can A, Celikkan F T, Cinar O. 2017. Umbilical cord mesenchymal stromal cell transplantations: A systemic analysis of clinical trials. Cytotherapy, 19(12): 1351-1382. doi:10.1016/j.jcyt.2017.08.004

Carvalho PH, Daibert F, Monteiro BS, Okano BS, Carvalho JL, Cunha D, Cunha LS, Favarato V, Pereira, Augusto L, Carlo R. 2013. Differentiation of adipose tissue-derived mesenchymal stem cells into cardiomyocytes. Arquivos Brasileiros de Cardiologia, 100(1): 82-89. doi:10.1590/s0066-782x2012005000114

Chen G, Yue A, Ruan Z, Yin Y, Wang R, Ren Y, Zhu L. 2015. Comparison of biological characteristics of mesenchymal stem cells derived from maternal-origin placenta and Whartons jelly. Stem Cell Research and Therapy, 6(1): 228-234. doi:10.1186/s13287-015-0219-6

Chen T M, Chen Y H, Sun H S, Tsai S J. 2019. Fibroblast growth factors: Potential novel targets for regenerative therapy of osteoarthritis. Chinese Journal of Physiology, 62(1): 2-10. doi:10.4103/CJP.CJP_11_19

Coutu DL, François M, Galipeau J. 2011. Inhibition of cellular senescence by developmentally regulated FGF receptors in mesenchymal stem cells. Blood, 117(25): 6801-6812. doi:

Cui Y, Ma S, Zhang C, Cao W, Liu M, Li D, Xing Q, Qu R, Yao, N. 2017. Human umbilical cord mesenchymal stem cells transplantation improves cognitive function in Alzheimers disease mice by decreasing oxidative stress and promoting hippocampal neurogenesis. Behavioural Brain Research, 320: 291-301. doi:10.1016/j.bbr.2016.12.021

Dermargos A, Armelin H. 2007. FGF-2: estudo de estrutura e função [Tesis de Doctorado]. [São Paulo (Brasil)], Univer- 
sidade de São Paulo.

Dolivo D, Hernandez S, Dominko T. 2016. Cellular lifespan and senescence: a complex balance between multiple cellular pathways. Bioessays, 38: S33-44. doi:10.1002/bies.201670906

Drela K, Sarnowska A, Siedlecka P, Szablowska-Gadomska I, Wielgos M, Jurga M, Lukomska B, Domanska-Janik K. 2014. Low oxygen atmosphere facilitates proliferation and maintains undifferentiated state of umbilical cord mesenchymal stem cells in an hypoxia inducible factor-dependent manner. Cytotherapy, 16(7): 881-892. doi:10.1016/j.jcyt.2014.02.009

El Agha E, Kosanovic D, Schermuly RT, Bellusci S. 2016. Role of fibroblast growth factors in organ regeneration and repair. In Seminars in Cell and Developmental Biology, 53: 76-84. doi:10.1016/j.semcdb.2015.10.009

Endo K, Fujita N, Nakagawa T, Nishimura R. 2019. Effect of fibroblast growth factor-2 and serum on canine mesenchymal stem cell chondrogenesis. Tissue Engineering Part A, 25(11-12): 901-910. doi:10.1089/ten.TEA.2018.0177

Eom YW, Oh JE, Lee JI, Baik SK, Rhee KJ, Shin HC, Kim C, Ahn J, Kong J, Shim K. 2014. The role of growth factors in maintenance of stemness in bone marrow-derived mesenchymal stem cells. Biochemical and Biophysical Research Communications, 445(1): 1622. doi:10.1016/j.bbrc.2014.01.084

Espinoza F, Aliaga F, Crawford PL. 2016. Escenario actual y perspectivas de la terapia con células madre mesenquimales en medicina intensiva. Revista Médica de Chile, 144(2): 222-231. doi:10.4067/S0034-98872016000200011

Fernandes-Freitas I, Owen BM. 2015. Metabolic roles of endocrine fibroblast growth factors. Current Opinion in Pharmacology, 25: 30-35. doi:10.1016/j.coph.2015.09.014

Guo YL, Chakraborty S, Rajan SS, Wang R, Huang F. 2010. Effects of oxidative stress on mouse embryonic stem cell proliferation, apoptosis, senescence, and selfrenewal. Stem Cells and Development, 19(9): 1321-1331. doi:10.1089/scd.2009.0313

Haghighi F, Dahlmann J, Nakhaei-Rad S, Lang A, Kutschka I, Zenker M, Kensah R, Piekorz R, Ahmadian MR. 2018. bFGF-mediated pluripotency maintenance in human induced pluripotent stem cells is associated with NRASMAPK signaling. Cell Communication and Signaling, 16(1): 96-109. doi:10.1186/s12964-018-0307-1

Hernández BM, Inostroza VC, Carrión AF, Chaparro PA, Quintero HA, Sanz RA. 2011. Proliferación de células madres mesenquimales obtenidas de tejido gingival humano sobre una matriz de quitosano: estudio in vitro. Revista Clínica de Periodoncia, Implantología y Rehabilitación Oral, 4(2): 59-63. doi:10.4067/S0719-01072011000200004

Hong SH, Lee MH, Koo MA, Seon GM, Park YJ, Kim D, Park JC. 2019. Stem cell passage affects directional migration of stem cells in electrotaxis. Stem Cell Research, 38: 101475. doi:10.1016/j.scr.2019.101475

Huang L, Wong YP, Gu H, Cai YJ, Ho Y, Wang CC, Leung T, Burd A. 2011. Stem cell-like properties of human umbilical cord lining epithelial cells and the potential for epidermal reconstitution. Cytotherapy, 13(2): 145-155. doi:10.3109/14653249.2010.509578

Ito T, Sawada R, Fujiwara Y, Seyama Y, Tsuchiya T.
2007. FGF-2 suppresses cellular senescence of human mesenchymal stem cells by down-regulation of TGF-2. Biochemical and Biophysical Research Communications, 359(1): 108-114. doi:10.1016/j.bbrc.2007.05.067

Katsares V, Petsa A, Felesakis A, Paparidis Z, Nikolaidou E, Gargani S, Karvounidou I, Ardelean K, Grigoriadis J. 2009. A rapid and accurate method for the stem cell viability evaluation: the case of the thawed umbilical cord blood. Laboratory Medicine, 40(9): 557-560. doi:10.1309/LMLE8BVHYWCT82CL

Korsensky L, Ron D. 2016. Regulation of FGF signaling: recent insights from studying positive and negative modulators. Seminars in Cell and Developmental Biology, 53: 101-114. doi:10.1016/j.semcdb.2016.01.023

Kurosu H, Ogawa Y, Miyoshi M, Yamamoto M, Nandi A, Rosenblatt KP, Baum MG, Schiavi S, Hu MC, Moe OW, Kuro-o M. 2006. Regulation of fibroblast growth factor-23 signaling by Klotho. The Journal of Biological Chemistry, 281: 6120-6123. doi:10.1074/jbc.C500457200

Lai WT, Krishnappa V, Phinney DG. 2011. Fibroblast growth factor 2 (Fgf2) inhibits differentiation of mesenchymal stem cells by inducing Twist2 and Spry4, blocking extracellular regulated kinase activation, and altering Fgf receptor expression levels. Stem Cells, 29(7): 1102-1111. doi:10.1002/stem.661

Lee JS, Kim SK, Jung BJ, Choi SB, Choi EY, Kim CS. 2018. Enhancing proliferation and optimizing the culture condition for human bone marrow stromal cells using hypoxia and fibroblast growth factor-2. Stem Cell Research, 28: 87-95. doi:10.1016/j.scr.2018.01.010

Markan KR, Potthoff MJ. 2016. Metabolic fibroblast growth factors (FGFs): mediators of energy homeostasis. Seminars in Cell and Developmental Biology, 53: 85-93. doi:10.1016/j.semcdb.2015.09.021

Meng X, Xue M, Xu P, Hu F, Sun B, Xiao Z. 2017. MicroRNA profiling analysis revealed different cellular senescence mechanisms in human mesenchymal stem cells derived from different origin. Genomics, 109(3-4): 147157. doi:10.1016/j.ygeno.2017.02.003

Naugler WE, Tarlow BD, Fedorov LM, Taylor M, Pelz C, Li B, Darnell J, Grompe M. 2015. Fibroblast growth factor signaling controls liver size in mice with humanized livers. Gastroenterology, 149(3): 728-740. doi:10.1053/j.gastro.2015.05.043

Nawrocka D, Kornicka K, Szydlarska J, Marycz K. 2017. Basic fibroblast growth factor inhibits apoptosis and promotes proliferation of adipose-derived mesenchymal stromal cells isolated from patients with type 2 diabetes by reducing cellular oxidative stress. Oxidative Medicine and Cellular Longevity, 2017: 3027109. doi:10.1155/2017/3027109

Novais A, Lesieur J, Sadoine J, Slimani L, Baroukh B, Saubaméa B, Schmitt A, Vital S, Rochefort GY. 2019. Priming Dental Pulp Stem Cells from Human Exfoliated Deciduous Teeth with Fibroblast Growth Factor2 Enhances Mineralization Within TissueEngineered Constructs Implanted in Craniofacial Bone Defects. Stem Cells Translational Medicine, 8(8): 844-857. doi:10.1002/sctm.18-0182

Nowwarote N, Pavasant P, Osathanon T. 2015. Role of endogenous basic fibroblast growth factor in 
stem cells isolated from human exfoliated deciduous teeth. Archives of Oral Biology, 60(3): 408-415. doi:10.1016/j.archoralbio.2014.11.017

Ornitz DM, Itoh N. 2001. Fibroblast growth factors. Genome Biology, 2(3): reviews3005.13005.12. doi:10.1186/gb2001-2-3-reviews:3005)

Park J, Lee JH, Yoon BS, Jun EK, Lee G, Kim IY, You S. 2018. Additive effect of bFGF and selenium on expansion and paracrine action of human amniotic fluid-derived mesenchymal stem cells. Stem Cell Research and Therapy, 9(1): 293-309. doi:10.1186/s13287-018-1058-z

Preda MB, Rosca AM, Tutuianu R, Burlacu A. 2015. Prestimulation with FGF-2 increases in vitro functional coupling of mesenchymal stem cells with cardiac cells. Biochemical and Biophysical Research Communications, 464(2): 667-673. doi:10.1016/j.bbrc.2015.07.055

Quimby JM, Borjesson DL. 2018. Mesenchymal stem cell therapy in cats: Current knowledge and future potential. Journal of Feline Medicine and Surgery, 20(3): 208-216. doi:10.1177/1098612X18758590

Richardson SM, Kalamegam G, Pushparaj PN, Matta C, Memic A, Khademhosseini A, Mobasheri R, Poletti F, Hoyland JA, Mobasheri A. 2016. Mesenchymal stem cells in regenerative medicine: focus on articular cartilage and intervertebral disc regeneration. Methods, 99: 69-80. doi:10.1016/j.ymeth.2015.09.015

Sah JP, Hao NTT, Kim Y, Eigler T, Tzahor E, Kim SH, Hwang Y, Yoon JK. 2019. MBP-FGF2-Immobilized Matrix Maintains Self-Renewal and Myogenic Differentiation Potential of Skeletal Muscle Stem Cells. International Journal of Stem Cells, 12(2):360-366. doi:10.15283/ijsc18125

Taupin P, Ray J, Fischer WH, Suhr ST, Hakansson K, Grubb A, Gage FH. 2000. FGF-2-responsive neural stem cell proliferation requires $\mathrm{CCg}$, a novel autocrine/paracrine cofactor. Neuron, 28(2): 385-397. doi:10.1016/S08966273(00)00119-7

Wang JJ, Liu YL, Sun YC, Ge W, Wang YY, Dyce PW, Hou R, Shen W. 2015. Basic fibroblast growth factor stimulates the proliferation of bone marrow mesenchymal stem cells in giant panda (ailuropoda melanoleuca). PloS One, 10(9): e0137712. doi:10.1371/journal.pone.0137712

Wang X, Ma S, Yang B, Huang T, Meng N, Xu L, Xing Q, Zhang Y, Li Q, Zhang T. 2018. Resveratrol promotes hUC-MSCs engraftment and neural repair in a mouse model of Alzheimers disease. Behavioural Brain Research, 339: 297-304. doi:10.1016/j.bbr.2017.10.032

Yang Y. 2018. Aging of mesenchymal stem cells: Implication in regenerative medicine. Regenerative Therapy, 9: 120-122. dpo:10.1016/j.reth.2018.09.002

Yonemitsu R, Tokunaga T, Shukunami C, Ideo K, Arimura H, Karasugi T, Nakamura J, Ide J, Hiraki Y, Mizuta H. 2019. Fibroblast Growth Factor 2 Enhances Tendon-toBone Healing in a Rat Rotator Cuff Repair of Chronic Tears. The American Journal of Sports Medicine, 47(7): 1701-1712. doi:10.1177/0363546519836959

Zhai W, Yong D, El-Jawhari JJ, Cuthbert R, Mcgonagle D, Naing MW, Jones E. 2019. Identification of senescent cells in multipotent mesenchymal stromal cell cultures: current methods and future directions. Cytotherapy, S1465-3249(19)30752-2. doi:10.1016/j.jcyt.2019.05.001
Zhang J, Li Y. 2016. Therapeutic uses of FGFs. Seminars in Cell and Developmental Biology, 53: 144-154. doi:10.1016/j.semcdb.2015.09.007

Zheng W, Nowakowski RS, Vaccarino FM. 2004. Fibroblast growth factor 2 is required for maintaining the neural stem cell pool in the mouse brain subventricular zone. Developmental Neuroscience, 26(2-4): 181-196. doi:10.1159/000082136

Ziaei M, Zhang J, Patel DV, McGhee CN. 2017. Umbilical cord stem cells in the treatment of corneal disease. Survey of Ophthalmology, 62(6): 803-815. doi:10.1016/j.survophthal.2017.02.002 